\title{
Geographic distance and amorphous iron affect the abundance and distribution of Geobacteraceae in paddy soils in China
}

\author{
Hai-Yan Yuan ${ }^{1,2}$ • Long-Jun Ding ${ }^{1}$. Ning Wang ${ }^{3} \cdot$ Song-Can Chen ${ }^{1,2} \cdot$ Ye Deng ${ }^{1,4}$. \\ Xiao-Ming $\mathrm{Li}^{1,2} \cdot$ Yong-Guan $\mathrm{Zhu}{ }^{1,5}$
}

Received: 15 April 2016 / Accepted: 19 May 2016 / Published online: 26 May 2016

(C) Springer-Verlag Berlin Heidelberg 2016

\begin{abstract}
Purpose Geobacteraceae are important dissimilatory Fe (III)reducing microorganisms, influencing the cycling of metals, nutrients as well as the degradation of organic contaminants. However, little is known about their distribution, diversity, and abundance of Geobacteraceae and the effects of environment factors and geographic distance on the distribution and diversity of Geobacteraceae in paddy soils remain unclear. Therefore, the objectives of this study were to investigate the distribution, diversity, and abundance of Geobacteraceae in paddy soils and to determine key factors in shaping the Geobacteraceae distribution, environmental factors,
\end{abstract}

Responsible editor: Zhihong Xu

Electronic supplementary material The online version of this article (doi:10.1007/s11368-016-1462-x) contains supplementary material, which is available to authorized users.

Yong-Guan Zhu

ygzhu@rcees.ac.cn

1 State Key Lab of Urban and Regional Ecology, Research Center for Eco-Environmental Sciences, Chinese Academy of Sciences, Beijing 100085, People's Republic of China

2 University of Chinese Academy of Sciences, Beijing 100049, People's Republic of China

3 Institute of Agricultural Resources and Environments, Jiangsu Academy of Agricultural Sciences, Nanjing 210014, People's Republic of China

4 CAS Key Laboratory of Environmental Biotechnology, Research Center for Eco-Environmental Sciences, Chinese Academy of Sciences, Beijing 100085, People's Republic of China

5 Key Lab of Urban Environment and Health, Institute of Urban Environment, Chinese Academy of Sciences, Xiamen 361021, People's Republic of China geographic distance, or both and to quantify their contribution to Geobacteraceae variation.

Materials and methods Illumina sequencing and quantitative real-time PCR using a primer set targeting $16 \mathrm{~S}$ rRNA genes of bacteria affiliated with the family Geobacteraceae were employed to measure the community composition, diversity, and abundance patterns of $16 \mathrm{~S}$ rRNA genes of Geobacteraceae in 16 samples collected from north to south of China. MRT, Mantel test, and VPA were used to analyze the relationship between communities of Geobacteraceae and environmental factors and geographic distance.

Results and discussion Quantitative PCR showed that the abundance of 16S rRNA genes of Geobacteraceae ranged from $(1.20 \pm 0.18) \times 10^{8}$ to $1.13 \times 10^{9} \pm 2.25 \times 10^{8}$ copies per gram of soil (dry weight) across different types of soils. Illumina sequencing results showed Geobacter was the dominant genus within the family of Geobacteraceae. Multivariate regression tree (MRT) analysis showed that soil amorphous iron contributed more $(22.46 \%)$ to the variation of dominant species of Geobacteraceae than other examined soil chemical factors such as $\mathrm{pH}(14.52 \%)$, ammonium (5.12\%), and dissolved organic carbon (4.74\%). Additionally, more geographically distant sites harbored less similar communities. Variance partitioning analysis (VPA) showed that geographic distance contributed more to the variation of Geobacteraceae than any other factor, although the environmental factors explained more variation when combined. So, we detected the uneven distribution of Geobacteraceae in paddy soils of China and demonstrated that Geobacteraceae community composition was strongly associated with geographic distance and soil chemical factors including aFe, $\mathrm{pH}$, $\mathrm{Fe}, \mathrm{DOC}, \mathrm{C}: \mathrm{N}$, and $\mathrm{NO}_{3}{ }^{-} \mathrm{N}$. These results greatly expand the knowledge of the distribution of Geobacteraceae in environments, particularly in terrestrial ecosystems. 
Conclusions Our results showed that geographic distance and amorphous iron played important roles in shaping Geobacteraceae community composition and revealed that both geographic distance and soil properties governed Geobacteraceae biogeography in paddy soils. Our findings will be critical in facilitating the prediction of element cycling by incorporating information on functional microbial communities into current biogeochemical models.

Keywords Amorphous iron (aFe) - Geobacteraceae . Illumina HiSeq sequencing · Iron reduction · Paddy soil

\section{Introduction}

Biogeochemical redox processes of iron $(\mathrm{Fe})$ are important in controlling the fate and behavior of contaminants and nutrients in the environment (Borch et al. 2009). Fe is the most abundant redox-active element on the Earth, and the vast majority is distributed as iron minerals in natural environments (Davison 1993; Stumm and Sulzberger 1992). Ferric iron (Fe (III)) and ferrous iron (Fe (II)) are the main redox states in the environments, and the transition between $\mathrm{Fe}$ (III) and $\mathrm{Fe}$ (II) is of particular interest in dynamic environments (Mejia et al. 2016). The microbially mediated redox cycling of Fe plays an important role in environmental biogeochemistry (Lalonde et al. 2012; Melton et al. 2014).

Paddy soil represents an intermediate between terrestrial and aquatic ecosystems and is usually employed as a model to study wetland biogeochemistry (Wang et al. 2015). Paddy soils experience regular and specific management practices such as submergence and drainage. The alternation between oxic and anoxic conditions results in periodically occurring redox reactions. Due to the unique characteristics of paddy soils and the great abundance of iron, dissimilatory Fe (III) reduction is prevalent and thought to be central to many other biogeochemical processes in paddy soils (Hori et al. 2015). It has been established that dissimilatory Fe (III) reduction strongly influences carbon $(\mathrm{C})$, nitrogen $(\mathrm{N})$, and sulfur (S) cycling and indirectly affects nutrient availability, greenhouse gas emissions, and contaminant transformation (Burgin et al. 2011; Coates et al. 1996; Hori et al. 2010; Yi et al. 2013).

Geobacteraceae represents a rare example of a family of microorganisms that play an important role in Fe redox processes in a diverse environment (Holmes et al. 2002). Most of the known dissimilatory $\mathrm{Fe}$ (III)-reducing microbes are in the family Geobacteraceae, which permits Geobacteraceae species to fill key niches in various anaerobic environments (Holmes et al. 2002; Snoeyenbos-West et al. 2000). However, previous studies on Geobacteraceae in paddy soils are mainly conducted in batch culture, in which microbes grow rapidly with high substrate concentrations (Yi et al. 2013). These conditions cannot reflect the natural conditions well, where microbes usually grow slowly due to nutrient limitation (Pierra et al. 2015). Meanwhile, those studies were conducted within an individual site, and it remains unclear how the distribution of Geobacteraceae is affected by geographic distance under field conditions. So far, our knowledge about the distribution and diversity of Geobacteraceae in paddy soils across a wide range of scales is limited, and the effects of geographic distance and environment variables on the distribution and diversity of Geobacteraceae in paddy soils remain unclear.

High-throughput sequencing technologies have opened new frontiers in microbial community analysis by providing a cost-effective means for identifying microbial phylotypes which are present in samples. Illumina HiSeq sequencing produces higher quality tags, greater throughput, and more userfriendly process compared with 454 pyrosequencing (Caporaso et al. 2010, 2012). Therefore, in our study, the distribution and diversity of Geobacteraceae in paddy soils under field conditions within a rather broad range spanning over $3500 \mathrm{~km}$ from north to south of China were examined by 16S rRNA gene-based Illumina HiSeq sequencing, and also the abundances of targeted microbes were analyzed by realtime PCR. The primary objectives of the present study were (i) to investigate the distribution, diversity, and abundance of Geobacteraceae in these soils and (ii) to determine key environment factors in shaping the Geobacteraceae distribution and quantify their contribution to Geobacteraceae variation.

\section{Materials and methods}

\subsection{Soil samples}

Soil samples were collected from various paddy soils at 16 different locations in China $\left(20^{\circ} 92^{\prime} \mathrm{N}\right.$ to $47^{\circ} 34^{\prime} \mathrm{N}$ and $105^{\circ}$ $27^{\prime} \mathrm{E}$ to $132^{\circ} 45^{\prime} \mathrm{E}$ ) in October 2014 (Table S1, Electronic Supplementary Material). The study area and sampling sites are presented in Fig. S1 (Electronic Supplementary Material). In each location, three soil cores $(5-\mathrm{cm}$ diameter and $10-\mathrm{cm}$ depth) were collected and mixed to form one composite sample. Soil samples were placed in sterile plastic bags, sealed, and transported to the laboratory on ice. Upon arrival at the laboratory, each soil sample was divided into two subsamples after homogenization. One subsample was stored at $4{ }^{\circ} \mathrm{C}$ in sealed containers for about 1 to 2 weeks before the soil physicochemical analysis, and the other was frozen at $-80{ }^{\circ} \mathrm{C}$ for the molecular analysis.

\subsection{Chemical analysis of soil samples}

Nitrate and ammonium were extracted with $2 \mathrm{M} \mathrm{KCl}$ and determined by a continuous flow analyzer (SAN++, Skalar, Holand). Soil $\mathrm{pH}$ was measured in a 1:2.5 soil:water $(w / v)$ 
suspension with a digital $\mathrm{pH}$ meter (PHS-3C, Shanghai Lida Instrument Company, China). The $\mathrm{C}: \mathrm{N}$ ratio was calculated based on the total $\mathrm{C}$ and total $\mathrm{N}$ contents that were measured on an element analyzer (Vario EL III, Elementar, Germany). The dissolved organic carbon (DOC) concentration was determined using total organic carbon (TOC) analyzer (Liquic TOC, Elementar, Germany). Total Fe and Mn contents were determined by an inductively coupled plasma optical emission spectrometer (ICP-OES, Optimal 2000 DV, Perkin-Elmer, USA). Amorphous iron (aFe) was extracted with oxalic acid described by Chao et al. (Chao and Zhou 1983). Soil electrical conductivity (EC) was determined by a portable conductivity meter (MP521, China).

\subsection{Genomic DNA extraction, PCR amplification, and community analysis}

Total DNA in the soil samples was extracted using the FastDNA SPIN kit for soils (MP Biomedicals, USA). The extracted DNA was visualized on a $1 \%$ agarose gel using ethidium bromide staining. DNA quality and quantity was assessed using a NanoDrop ND-2000 spectrophotometer (NanoDrop Co., USA).

PCR was conducted in triplicate to detect the Geobacteraceae 16S rRNA genes, using the primers Geo494F (5'-barcode AGG AAG CAC CGG CTA ACT CC-3') and Geo825R (5'-TAC CCG CRA CAC CTA GT-3') with sample-identifying barcodes (Holmes et al. 2002). PCR amplifications were performed in $50 \mu \mathrm{l}$ reaction mixtures containing $\sim 40 \mathrm{ng}$ of template DNA, $25 \mu \mathrm{l}$ Dream Taq Green PCR master mix $(2 \times), 20.5 \mu \mathrm{H} \mathrm{H}_{2} \mathrm{O}, 0.5 \mu \mathrm{l} 1 \%$ bovine serum albumin (BSA), and $0.2 \mu \mathrm{M}$ each primer. The thermal conditions of PCR were as follows: initial denaturation at $94{ }^{\circ} \mathrm{C}$ for $5 \mathrm{~min} ; 30$ cycles of denaturation at $94{ }^{\circ} \mathrm{C}$ for $30 \mathrm{~s}$, annealing at $51{ }^{\circ} \mathrm{C}$ for $30 \mathrm{~s}$, and extension at $72{ }^{\circ} \mathrm{C}$ for $30 \mathrm{~s}$ and the final extension step at $72{ }^{\circ} \mathrm{C}$ for $7 \mathrm{~min}$. The amplicons were pooled from the triplicate reactions, purified using the universal DNA purification kit (Tiangen, China), and normalized in equimolar amounts. Finally, the PCR amplicons were submitted to Novogene Bioinformatics Institute (Beijing, China) for sequencing on the Illumina HiSeq 2500 platform.

Raw reads with contaminated adapters, or an undetermined nucleotide $(\mathrm{N})$, or one base occurring more than 10 times successively were removed. Low-quality reads (containing $>20$ low-quality bases) were further filtered from the data, and then primer regions were removed. The above processes were conducted by Novogene Bioinformatics Institute in a pre-bioinformatics analysis. The resulted sequences were subsequently analyzed using Quantitative Insights Into Microbial Ecology (QIIME) (version 1.6) according to a previous study (Caporaso et al. 2010). Operational taxonomic units (OTUs) were picked at $97 \%$ sequences similarity level on the basis of an open reference by searching reads against the Greengenes database. Representative sequence for each OTU was then chosen for subsequent alignment and taxonomic assignment with the RDP classifier. Chimeric sequences, chloroplast and mitochondrial OTUs (around $1 \%$ ), and singleton OTUs were discarded from the final OTU table. OTUs with the same taxonomy were combined at different taxonomic levels. Both $\alpha$-diversity (including Shannon, Simpson, and Phylogenetic diversity (PD)) and $\beta$-diversity calculations were performed based on the OTU table with a random sampling depth of the minimal sequencing number (11919) among samples. For $\beta$-diversity analysis, dissimilarity of Geobacteraceae was calculated using principal-coordinate analysis (PCoA) on pairwise, weighted UniFrac distances among all samples. Additionally, PCoA based on pairwise Bray-Curtis dissimilarity was performed using QIIME.

\subsection{Quantitative real-time PCR for 16S rRNA gene sequences of Geobacteraceae and bacteria}

The abundance of Geobacteraceae bacteria in the paddy soils was evaluated by quantitative PCR (qPCR) assay using a primer set targeting $16 \mathrm{~S}$ rRNA genes of bacteria affiliated with the family Geobacteraceae: Geo494F and Geo825R (Holmes et al. 2002). qPCR was performed on an iQTM5 Thermocycler (Bio-Rad, USA) in $25 \mu$ l-reaction mixtures containing $12.5 \mu \mathrm{l}$ SYBR Premix Ex TaqTM (Takara Bio Inc., Japan), $1 \mu$ l DNA template, and $1 \mu$ of each $10 \mu \mathrm{M}$ primers. As thermal conditions, after an initial denaturation at $94{ }^{\circ} \mathrm{C}$ for $5 \mathrm{~min}$, targeted DNA was amplified in 35 cycles; each cycle consisted of denaturation for $30 \mathrm{~s}$ at $94{ }^{\circ} \mathrm{C}$, annealing for $30 \mathrm{~s}$ at $51{ }^{\circ} \mathrm{C}$, and extension for $40 \mathrm{~s}$ at $72{ }^{\circ} \mathrm{C}$. Fluorescence was collected at the end of the extension step. Following PCR, amplicons were assessed by a melting-curve analysis to check for successful amplification.

The standard template DNA was prepared using the primer pair Geo494F/Geo825R to amplify $16 \mathrm{~S}$ rRNA genes of Geobacteraceae. The PCR amplicons were ligated to pGEM-T Easy vector (Promega, USA) and transformed into Escherichia coli JM109 cells. The positive clones with the targeted gene insert were sequenced, and the most abundant one was used for plasmid DNA extraction. After concentration measurement with Nanodrop ND-2000 UV-vis spectrophotometer (NanoDrop Co., USA), the purified plasmid DNA was serially tenfold diluted and subjected to qPCR in triplicate to generate an external standard curve. The abundance of $16 \mathrm{~S}$ rRNA genes was estimated using a TaqMan assay with the primer set $1369 \mathrm{~F} / 1492 \mathrm{R}$ and a probe $1389 \mathrm{~F}$ as described previously (Zhang et al. 2015). Target gene copy numbers were calculated from the standard curves and presented per gram of dry weight of soil. Moreover, the relative abundance of Geobacteraceae DNA to the total extracted DNA was also determined. 


\subsection{Statistical analysis}

All statistical analyses were conducted using QIIME (http:// qiime.sourceforge.net/), R (http://www.r-project.org/) and SPSS (SPSS statistics 17.0). Nonmetric multidimensional scaling (NMDS) was performed to cluster the different sites by their soil physicochemical similarities. Spearman correlation coefficient was used to test the relationships between soil physicochemical parameters and the abundance and diversity indices of Geobacteraceae. Multivariate regression tree (MRT) was used to illustrate the community dissimilarities among different paddy soil samples. MRT is a constrained classification approach which is well suited for ecological data sets and has been successfully used for exploring key environmental variables that influence microbial communities (Ge et al. 2012). Heatmap was generated using the software of HemI (HemI 1.0, Heatmap IIIustrator). The OTUs with relative abundances $>1 \%$ (the percentage of each OTU to total OTUs) were normalized and used to generate heatmap. The pairwise geographic distances between different paddy soil sites were calculated based on geographic coordinates. The relationships between the similarity of community (taxonomic similarity and phylogenetic similarity) and geographical distance were measured by linear models. The taxonomic community similarity was based on the Bray-Curtis distance and the phylogenetic community similarity was based on weighted UniFrac distance (Rodrigues et al. 2013). Partial Mantel test was performed to analyze the relationships between communities of Geobacteraceae and soil variables or spatial patterns, using Euclidean distance. BioEnv and canonical correspondence analysis (CCA) were also used to identify the abiotic factors most important to the composition of Geobacteraceae, and they were used to construct the soil property matrix for variance partitioning analysis (VPA).

\section{Results}

\subsection{Physicochemical properties of the soil samples}

Physicochemical properties of paddy soils are listed in Table 1. Soil characteristics varied considerably with sampling sites. Concentration of aFe ranged from 2.16 to $19.86 \mathrm{~g} \mathrm{~kg}^{-1}$. Concentrations of total $\mathrm{Fe}$ and $\mathrm{Mn}$ ranged from 17.04 to $41.43 \mathrm{~g} \mathrm{~kg}^{-1}$ and 0.16 to $0.97 \mathrm{~g} \mathrm{~kg}^{-1}$, respectively. Concentrations of dissolved organic carbon (DOC), ammonium- $\mathrm{N}\left(\mathrm{NH}_{4}{ }^{+}-\mathrm{N}\right)$, and nitrate- $\mathrm{N}\left(\mathrm{NO}_{3}{ }^{-}-\mathrm{N}\right)$ were highly variable across the samples, averaging at $232.62 \pm 93.90$, $12.01 \pm 3.51,17.37 \pm 6.64 \mathrm{mg} \mathrm{kg}^{-1}$, respectively. Soil $\mathrm{pH}$ $(5.58 \pm 1.13)$ and $\mathrm{C}: \mathrm{N}$ ratio $(11.10 \pm 1.85)$ were also subjected to considerable fluctuations. Concentrations of EC ranged from 360 to $2527 \mu \mathrm{s} \mathrm{cm}^{-1}$. Additionally, NMDS analysis also confirmed the high heterogeneity among the paddy soils (Fig. S2, Electronic Supplementary Material).

\subsection{Abundance of Geobacteraceae}

The abundance of Geobacteraceae was estimated on the basis of quantification of 16S rRNA genes of the Geobacteraceae using qPCR. The copy numbers of Geobacteraceae in soil samples ranged from $1.20 \times 10^{8} \pm 1.85 \times 10^{7}$ to $1.13 \times 10^{9} \pm 2.25 \times 10^{8}$ copies per gram of dry weight (Fig. 1 and Fig. S3, Electronic Supplementary Material). The relative abundance of Geobacteraceae in paddy soil samples ranged from 0.27 to $9.90 \%$ (Fig. 1 and Fig. S4, Electronic Supplementary Material). Spearman correlation analysis showed that the abundance of Geobacteraceae was significantly correlated with $\mathrm{Fe}$ and aFe (Table S2, Electronic Supplementary Material). Moreover, spearman correlation analysis indicated that the relative abundance of Geobacteraceae was significantly correlated with the DOC, $\mathrm{NH}_{4}{ }^{+}-\mathrm{N}$, latitude, longitude, $\mathrm{Fe}$, and aFe (Table S3, Electronic Supplementary Material).

\subsection{Community composition and diversity of Geobacteraceae}

A total of 572,112 high-quality sequences of Geobacteraceae were obtained, and all of them were assigned to 2908 operational taxonomic units (OTUs) at a $97 \%$ similarity level. The community structure of Geobacteraceae was dominated by the genus Geobacter (more than $99 \%$ of the total sequences). Additionally, $0.19 \%$ sequences belonged to the genus Geopsychrobacter and $0.60 \%$ to the genus Geothermobacter. The diversity of the Geobacteraceae was highly variable across the 16 samples, with higher diversity in JSJ, SY, MY, JS, JX, JL, and AX (Simpson index ranged from 100.86 to 113.81 ) and the lowest diversity in DH (Table S4, Electronic Supplementary Material). Spearman correlation analysis showed that the Shannon and Simpson estimators did not significantly correlate with any examined environmental factors, while PD was significantly related with DOC, $\mathrm{NH}_{4}{ }^{+} \mathrm{N}$, and $\mathrm{NO}_{3}{ }^{-}-\mathrm{N}$ (Table S5, Electronic Supplementary Material).

\subsection{Relationships of community composition of Geobacteraceae to soil chemical properties and geographical distance}

We used MRT to identify the relative importance of soil variables contributing to the distribution of main species of Geobacteraceae in different paddy samples. The main species were composed of OTUs detected in every sample, making up 89 to $92 \%$ of the total reads in these soil samples (Table S6, Electronic Supplementary Material). MRT analysis showed a 
Table 1 Physicochemical properties of the 16 paddy soils

\begin{tabular}{|c|c|c|c|c|c|c|c|c|c|}
\hline \multirow[t]{2}{*}{ Sample } & \multirow[t]{2}{*}{$\mathrm{pH}$} & \multicolumn{3}{|c|}{$\mathrm{mg} \mathrm{kg}^{-1}$ dry paddy soil } & \multicolumn{3}{|c|}{$\mathrm{g} \mathrm{kg}^{-1}$ dry paddy soil } & \multirow[b]{2}{*}{$\mathrm{C}: \mathrm{N}$} & \multirow{2}{*}{$\begin{array}{l}\mu \mathrm{s} \mathrm{cm}{ }^{-1} \\
\mathrm{EC}\end{array}$} \\
\hline & & DOC & $\mathrm{NH}_{4}{ }^{+}-\mathrm{N}$ & $\mathrm{NO}_{3}{ }^{-}-\mathrm{N}$ & $\mathrm{Fe}$ & $\mathrm{aFe}$ & $\mathrm{Mn}$ & & \\
\hline HL & $6.58 \pm 0.58$ & $210.56 \pm 6.20$ & $20.42 \pm 3.38$ & $8.81 \pm 0.38$ & $30.68 \pm 2.49$ & $9.65 \pm 0.28$ & $0.93 \pm 0.37$ & $12.48 \pm 0.29$ & $594 \pm 82$ \\
\hline JSJ & $5.89 \pm 0.24$ & $181.27 \pm 6.76$ & $26.11 \pm 1.34$ & $14.11 \pm 0.66$ & $23.07 \pm 1.21$ & $16.71 \pm 1.69$ & $0.97 \pm 0.22$ & $10.78 \pm 0.12$ & $452 \pm 99$ \\
\hline $\mathrm{BC}$ & $8.06 \pm 0.15$ & $113.49 \pm 5.39$ & $17.26 \pm 4.54$ & $12.05 \pm 0.78$ & $21.69 \pm 1.88$ & $2.17 \pm 0.27$ & $0.36 \pm 0.04$ & $10.67 \pm 0.79$ & $1406 \pm 39$ \\
\hline WC & $6.29 \pm 0.64$ & $140.52 \pm 4.09$ & $21.77 \pm 2.34$ & $9.94 \pm 0.68$ & $29.40 \pm 2.50$ & $14.69 \pm 0.11$ & $0.66 \pm 0.05$ & $11.93 \pm 0.12$ & $494 \pm 75$ \\
\hline $\mathrm{DH}$ & $7.85 \pm 0.34$ & $130.64 \pm 6.02$ & $16.01 \pm 5.06$ & $12.62 \pm 0.97$ & $32.74 \pm 1.88$ & $12.46 \pm 0.47$ & $0.79 \pm 0.21$ & $11.69 \pm 0.06$ & $2391 \pm 412$ \\
\hline SY & $6.67 \pm 0.62$ & $104.85 \pm 8.11$ & $9.84 \pm 1.91$ & $8.81 \pm 0.34$ & $28.82 \pm 2.91$ & $5.88 \pm 0.32$ & $0.46 \pm 0.10$ & $11.14 \pm 0.03$ & $821 \pm 252$ \\
\hline MY & $7.92 \pm 0.04$ & $354.35 \pm 11.52$ & $10.05 \pm 0.85$ & $12.55 \pm 0.05$ & $20.63 \pm 0.98$ & $4.48 \pm 0.13$ & $0.45 \pm 0.02$ & $14.89 \pm 0.09$ & $2527 \pm 49$ \\
\hline JS & $5.72 \pm 0.30$ & $224.13 \pm 6.02$ & $6.73 \pm 0.42$ & $20.58 \pm 0.46$ & $32.35 \pm 0.12$ & $7.62 \pm 0.16$ & $0.40 \pm 0.01$ & $10.52 \pm 0.06$ & $623 \pm 31$ \\
\hline JX & $6.27 \pm 0.22$ & $211.20 \pm 5.18$ & $26.22 \pm 5.82$ & $9.39 \pm 0.54$ & $35.71 \pm 0.42$ & $13.6 \pm 0.16$ & $0.66 \pm 0.04$ & $8.57 \pm 0.07$ & $629 \pm 148$ \\
\hline $\mathrm{AH}$ & $5.22 \pm 0.03$ & $251.34 \pm 7.54$ & $16.70 \pm 4.83$ & $9.08 \pm 0.69$ & $41.43 \pm 1.30$ & $16.89 \pm 0.85$ & $0.56 \pm 0.01$ & $9.48 \pm 0.07$ & $360 \pm 63$ \\
\hline $\mathrm{JL}$ & $7.93 \pm 0.17$ & $248.15 \pm 6.20$ & $12.74 \pm 4.54$ & $17.88 \pm 3.90$ & $29.47 \pm 2.08$ & $7.63 \pm 0.03$ & $0.56 \pm 0.05$ & $15.32 \pm 0.03$ & $1725 \pm 559$ \\
\hline $\mathrm{AX}$ & $7.93 \pm 0.02$ & $250.60 \pm 11.84$ & $10.94 \pm 2.15$ & $14.59 \pm 0.25$ & $40.02 \pm 0.48$ & $12.03 \pm 0.8$ & $0.86 \pm 0.00$ & $11.19 \pm 0.01$ & $2036 \pm 186$ \\
\hline TY & $5.02 \pm 0.05$ & $278.16 \pm 4.98$ & $19.30 \pm 4.12$ & $9.93 \pm 0.57$ & $20.33 \pm 0.62$ & $2.61 \pm 0.09$ & $0.21 \pm 0.02$ & $9.01 \pm 0.10$ & $615 \pm 86$ \\
\hline YT & $5.22 \pm 0.07$ & $484.85 \pm 22.13$ & $22.84 \pm 4.94$ & $9.76 \pm 0.52$ & $17.04 \pm 0.45$ & $2.64 \pm 0.02$ & $0.16 \pm 0.01$ & $10.21 \pm 0.95$ & $553 \pm 46$ \\
\hline BJ & $7.40 \pm 0.85$ & $308.53 \pm 6.88$ & $15.83 \pm 1.97$ & $13.29 \pm 2.01$ & $36.79 \pm 9.32$ & $19.86 \pm 1.38$ & $0.70 \pm 0.32$ & $10.16 \pm 0.03$ & $1723 \pm 552$ \\
\hline $\mathrm{LZ}$ & $5.32 \pm 0.04$ & $229.30 \pm 5.30$ & $25.11 \pm 2.99$ & $8.79 \pm 0.14$ & $23.70 \pm 0.63$ & $16.22 \pm 0.7$ & $0.18 \pm 0.00$ & $9.48 \pm 0.04$ & $419 \pm 26$ \\
\hline
\end{tabular}

nested structure, with communities splitting first by aFe, then by $\mathrm{pH}, \mathrm{aFe}$ (on the left split), $\mathrm{NH}_{4}{ }^{+}-\mathrm{N}$, and DOC (Fig. 2). aFe explained $22.46 \%$ of the variation in Geobacteraceae community composition. $\mathrm{pH}$ explained $14.52 \%$ and aFe (on the left split) explained $5.58 \%$. Then, $\mathrm{NH}_{4}{ }^{+}-\mathrm{N}$ and DOC explained 5.12 and $4.74 \%$, respectively. Heatmap showed the dominant species of Geobacteraceae in these paddy soils (Table S6, Electronic Supplementary Material). Patterns of each OTU in six splits were different, which illustrated the effect of aFe, $\mathrm{pH}, \mathrm{NH}_{4}{ }^{+}-\mathrm{N}$, and DOC on the distribution of the main species of Geobacteraceae. Together, these results suggested that aFe was an important indicator for the distribution of the dominant species of Geobacteraceae in paddy soils of China.

The partial Mantel test revealed a significant correlation between the Geobacteraceae communities and the
Fig. 1 Abundance and relative abundance of Geobacteraceae in paddy soils in China. The base map used is from the National Fundamental Geographic Information System of China. The red and green columns represent estimated abundance and relative abundance of Geobacteraceae, respectively

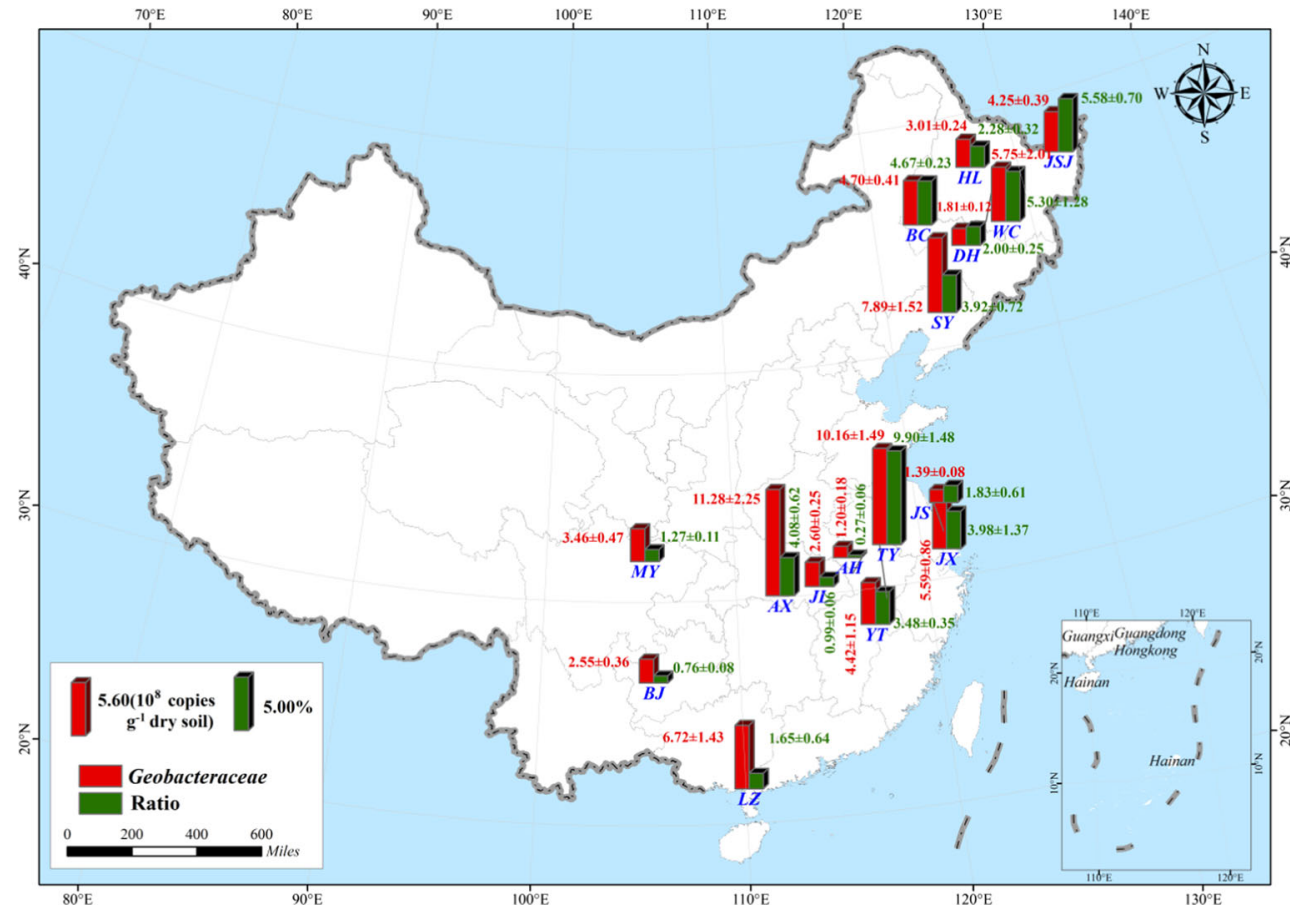


Fig. 2 Multivariate regression tree (MRT) to reveal the hierarchical environmental determinants on communities of Geobacteraceae with amorphous iron $(\mathrm{aFe}), \mathrm{pH}$, amorphous iron $(\mathrm{aFe}), \mathrm{NH}_{4}-\mathrm{N}$, and DOC. In an MRT, each spit is represented graphically as a branch that is labeled with the levels of the classification variable; bar plots show the multivariate means of OTUs at each branch; the numbers of samples included in the splits are shown under the bar plot. The heatmap shows the normalized abundances of OTUs (the OTUs higher than $1 \%$ percentage) that occurred in 48 samples

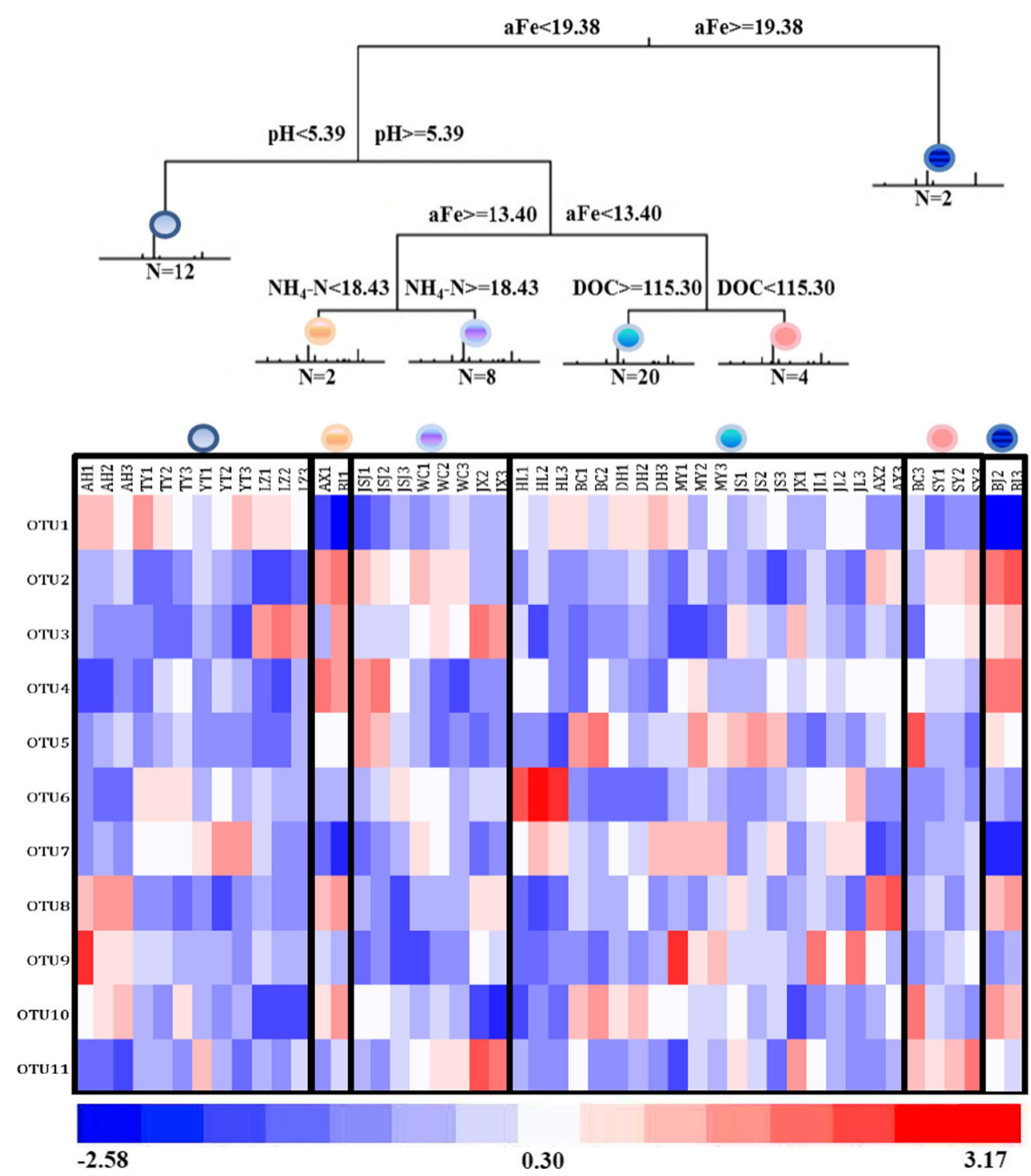

environmental factors including $\mathrm{Fe}$, aFe, Mn, latitude, and longitude (Table 2). Due to the significant correlation between Geobacteraceae communities and latitude or longitude, we linked the communities of Geobacteraceae with geographic distance. The relationships were calculated by determining the slope between geographic distance and community similarity of Geobacteraceae. It showed that both taxonomy similarity (Bray-Curtis) and phylogenetic similarity (weighted UniFrac) were significantly correlated with geographic distance. Plots of taxonomic similarity $\left(R^{2}=0.0301, P=0.032\right)$ or phylogenetic similarity $\left(R^{2}=0.052, P=0.007\right)$ versus geographic distance for each pairwise set of samples revealed that the Geobacteraceae displayed a significant, negative distancedecay curve across the scales from $92 \mathrm{~km}$ up to approximately 3515 km (Fig. 3).

VPA was performed to quantify the relative contribution of geographic distance and soil properties to the taxonomic structure of Geobacteraceae. A subset of environmental
Table 2 Mantel analysis of the relationship between the communities of Geobacteraceae and soil properties

\begin{tabular}{llll}
\hline Family & Variable & $r$ & $P$ \\
\hline Geobacteraceae & $\mathrm{pH}$ & 0.039 & 0.128 \\
& $\mathrm{DOC}$ & -0.009 & 0.442 \\
& $\mathrm{NH}_{4}{ }^{+}-\mathrm{N}$ & -0.059 & 0.868 \\
$\mathrm{NO}^{-}{ }^{-} \mathrm{N}$ & -0.026 & 0.535 \\
& $\mathrm{C}: \mathrm{N}$ & -0.079 & 0.867 \\
$\mathrm{EC}$ & 0.040 & 0.249 \\
& $\mathrm{Mn}$ & 0.132 & $\mathbf{0 . 0 5 0}$ \\
& $\mathrm{Fe}$ & 0.129 & $\mathbf{0 . 0 4 5}$ \\
& aFe & 0.184 & $\mathbf{0 . 0 0 9}$ \\
& Latitude & 0.092 & $\mathbf{0 . 0 5 0}$ \\
& Longitude & 0.251 & $\mathbf{0 . 0 0 1}$ \\
\hline
\end{tabular}

Significant $(P \leq 0.05)$ values are presented in bold 

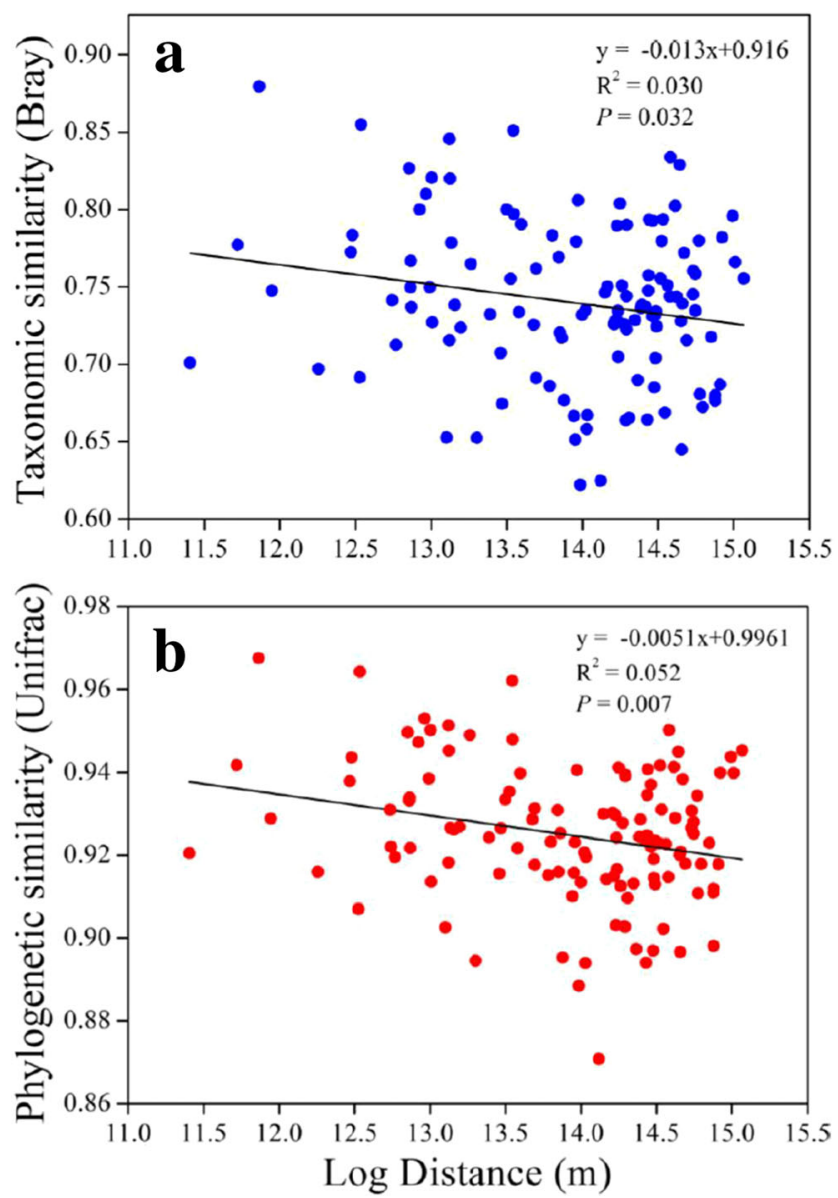

Fig. 3 The relationship between the beta-diversity with geographic distance. a Decay of taxonomic similarity (Bray-Curtis) with geographic distance. b Decay of phylogenetic similarity (weighted UniFrac) with geographic distance

parameters (aFe, $\mathrm{pH}, \mathrm{Fe}, \mathrm{DOC}, \mathrm{C}: \mathrm{N}$, and $\left.\mathrm{NO}_{3}{ }^{-} \mathrm{-N}\right)$ was selected by the BioEnv procedure, which provides the highest Pearson correlation with Geobacteraceae. These variables explained $23.3 \%$ of the observed variation, leaving $76.6 \%$ of the variation unexplained. The soil properties explained $18.2 \%$ and geographic distance explained $5.1 \%$ variations, and no interaction effect was detected. Although the soil properties explained more of the variation, geographic distance by itself explained $5.1 \%$ of the variation observed, more than any of the other 6 of the individual soil properties (Fig. 4). Thus, both soil properties and geographic distance are important factors in shaping the structure of Geobacteraceae.

\section{Discussion}

In the present study, the copy numbers of Geobacteraceae and the relative abundances of Geobacteraceae in paddy soil samples varied greatly among soil samples. We detected the uneven distribution of Geobacteraceae in paddy soils of China and demonstrated that Geobacteraceae community composition was

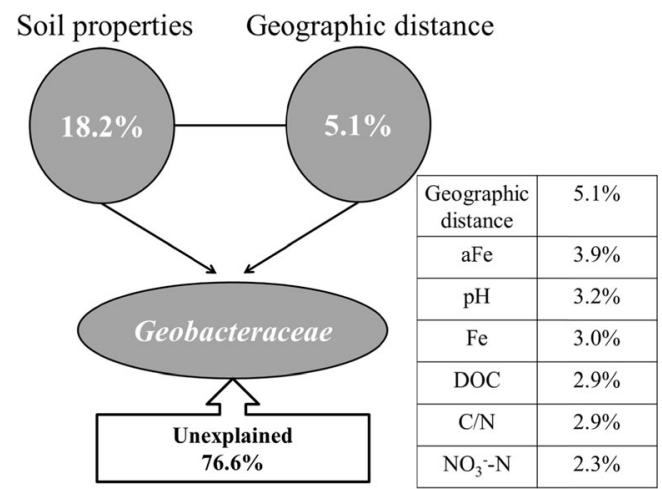

Fig. 4 Variance partition analysis of the effects of geographic distance and soil properties on the phylogenetic structure of Geobacteraceae

strongly associated with soil chemical factors including aFe, $\mathrm{pH}, \mathrm{Fe}, \mathrm{DOC}, \mathrm{C}: \mathrm{N}$, and $\mathrm{NO}_{3}{ }^{-}-\mathrm{N}$ and geographic distance. These results greatly expand the knowledge of the distribution of Geobacteraceae in environments, particularly in terrestrial ecosystems.

Environmental heterogeneity and geographic distance are considered to be major factors driving current differences in microbial diversity (Liu et al. 2015). Our results showed that the 16S rRNA genes of Geobacteraceae exhibited a biogeographic pattern that agreed with previous work on the biogeography of soil bacterial communities in soils (Ge et al. 2008; Griffiths et al. 2011). We can consider our results within the framework of factors that drive the biogeography of microbial communities, including selection, drift, dispersal, and mutation (Hanson et al. 2012). Selection and dispersal both have the effect on increasing distinctiveness of a community and push distance-decay slopes more negative. Meanwhile, endemism is a widely recognized phenomenon in microbial ecology and could be facilitated by the broad dissimilarities of soil physicochemical properties and climate conditions that serve as clear barriers to dispersal in many locations, which indicated that geographic distance and environmental selection and dispersal may act on the 16S rRNA genes of Geobacteraceae (Jiang et al. 2016).

It is interesting to note that aFe explained more than the other examined soil physicochemical parameters in the distribution of Geobacteraceae especially the dominant species of Geobacteraceae in paddy soils. aFe can be used as an electron acceptor to facilitate the respiration of Geobacteraceae (Lovley et al. 2004). Previous studies have showed that higher level of aFe favored the growth of iron-reducing bacteria, especially the growth of Geobacteraceae species (Ding et al. 2015; Kato et al. 2010).

$\mathrm{Fe}$ (III) reduction couples the $\mathrm{Fe}, \mathrm{C}, \mathrm{S}, \mathrm{P}$, and $\mathrm{N}$ cycles and occurs in paddy soils (Melton et al. 2014). For example, Geobacteraceae could inhibit methane production (Jäckel et al. 2005). The suppression of methanogenesis is mostly from out-competition of methanogens by iron-reducing microorganisms for common electron donors (Hori et al. 
2010). The availability of iron for microbial reduction is an important factor controlling the extent of organic matter decomposition, with $\mathrm{Fe}$ (III) serving as the terminal electron acceptor (Lovley 1987). Geobacteraceae can metabolize organic matter that otherwise would be metabolized by methanogenic organisms in paddy soils (Deng et al. 2015). Due to the importance of dissimilatory Fe (III) reduction to many other biogeochemical processes, our findings have far-reaching implications for understanding of iron cycling in paddy soils and its impacts on the environment.

In addition to aFe, our results also showed that the community structure of Geobacteraceae was significantly correlated with soil $\mathrm{pH}$. Previous studies have demonstrated that soil $\mathrm{pH}$ is a major factor in determining the soil bacterial biodiversity and composition. For instance, soil $\mathrm{pH}$ has been shown to influence bacterial communities in British soils (Griffiths et al. 2011), Arctic soils (Chu et al. 2010), soils across North and South American (Lauber et al. 2009), and soils of Northeast China (Liu et al. 2014). pH seems to be a key variable in the soil environment (Feng et al. 2014). Fe (III) primarily exists as insoluble, solid-phase minerals in circumneutral $\mathrm{pH}$ environment. The evidence suggested that Fe (III) oxides provide an electron sink and are reduced biologically in neoteric environmental systems in the anoxic zone at $\mathrm{pH}>4.0$ (Weber et al. 2006). Therefore, soil $\mathrm{pH}$ is a reasonable predictor of $\mathrm{Fe}$ (III)reducing microorganisms at the continental scale. In the current study, $\mathrm{pH}$ was also a predictor of the community structure of Geobacteraceae.

This is the first study to investigate the distribution of Geobacteraceae by Illumina HiSeq sequencing. It has been suggested that genomics data can be integrated into biogeochemical model to predict environmental changes modulated by diverse functional microbes (Reed et al. 2014). The importance of microbe-mediated iron redox changes in controlling the biogeochemistry of elements in the environment, particularly in sediments and wetland, is well established (Melton et al. 2014), Paddy soils are subjected to regular dry-wet cycles, and longterm paddy cultivation will lead to the accumulation of amorphous iron, which is a common terminal electron acceptor (Ding et al. 2015; Li et al. 2011). Therefore, understanding the diversity and distribution of Geobacteraceae, a major group of microbes involved in dissimilatory iron reducers in paddy soils, at large scale will be indispensible in predicting the biogeochemical cycling of contaminants and nutrients. Furthermore, our findings have implications for understanding the ecology of Geobacteraceae in terrestrial system and will provide information for predicting how the community of Geobacteraceae will respond to environmental perturbations, such as climate change.

\section{Conclusions}

In summary, this study gives insight into how geographic distance and environmental factors structure the community of Geobacteraceae within a rather broad range spanning over $3500 \mathrm{~km}$ from north to south of China. We reveal that the community composition of Geobacteraceae is modulated by geographic distance and soil properties. This is the first study to demonstrate that geographic distance can predict community structure of Geobacteraceae across a large scale. Moreover, aFe as a factor is also a contributor to community variation of Geobacteraceae especially the dominant species of Geobacteraceae in paddy soils. These results can potentially be integrated into current biogeochemical models in better predicting the fate and behavior of elements in terrestrial environments.

Acknowledgments This work was supported by the National Natural Science Foundation of China (41430858) and the Strategic Priority Research Program of Chinese Academy of Sciences (XDB15020402).

\section{Compliance with ethical standards}

Conflict of interest The authors declare that they have no competing interests.

\section{References}

Borch T, Kretzschmar R, Kappler A, Cappellen PV, Ginder-Vogel M, Voegelin A, Campbell K (2009) Biogeochemical redox processes and their impact on contaminant dynamics. Environ Sci Technol 44(1):15-23

Burgin AJ, Yang WH, Hamilton SK, Silver WL (2011) Beyond carbon and nitrogen: how the microbial energy economy couples elemental cycles in diverse ecosystems. Front Ecol Environ 9(1):44-52

Caporaso JG, Kuczynski J, Stombaugh J, Bittinger K, Bushman FD, Costello EK, Fierer N, Pena AG, Goodrich JK, Gordon JI (2010) QIIME allows analysis of high-throughput community sequencing data. Nat Methods 7(5):335-336

Caporaso JG, Lauber CL, Walters WA, Berg-Lyons D, Huntley J, Fierer N, Owens SM, Betley J, Fraser L, Bauer M (2012) Ultra-highthroughput microbial community analysis on the Illumina HiSeq and MiSeq platforms. ISME J 6(8):1621-1624

Chao T, Zhou L (1983) Extraction techniques for selective dissolution of amorphous iron oxides from soils and sediments. Soil Sci Soc Am J 47:225-232

Chu H, Fierer N, Lauber CL, Caporaso J, Knight R, Grogan P (2010) Soil bacterial diversity in the Arctic is not fundamentally different from that found in other biomes. Environ Microbiol 12(11):2998-3006

Coates JD, Phillips E, Lonergan DJ, Jenter H, Lovley DR (1996) Isolation of Geobacter species from diverse sedimentary environments. Appl Environ Microbiol (AEM) 62(5):1531-1536

Davison W (1993) Iron and manganese in lakes. Earth-Sci Rev 34(2):119-163

Deng D, Zhang Y, Liu Y (2015) A Geobacter strain isolated from rice paddy soil with higher bioelectricity generation capability in comparison to Geobacter sulfurreducens PCA. RSC Adv 5(55):4397843989 
Ding L-J, Su J-Q, Xu H-J, Jia Z-J, Zhu Y-G (2015) Long-term nitrogen fertilization of paddy soil shifts iron-reducing microbial community revealed by $\mathrm{RNA}-{ }^{13} \mathrm{C}$-acetate probing coupled with pyrosequencing. ISME J 9(3):721-734

Feng Y, Grogan P, Caporaso JG, Zhang H, Lin X, Knight R, Chu H (2014) $\mathrm{pH}$ is a good predictor of the distribution of anoxygenic purple phototrophic bacteria in Arctic soils. Soil Biol Biochem 74:193-200

Ge Y, He J-z, Zhu Y-g, Zhang J-b, Xu Z, Zhang L-m, Zheng Y-m (2008) Differences in soil bacterial diversity: driven by contemporary disturbances or historical contingencies? ISME J 2(3):254-264

Ge Y, Schimel JP, Holden PA (2012) Identification of soil bacteria susceptible to $\mathrm{TiO}_{2}$ and $\mathrm{ZnO}$ nanoparticles. Appl Environ Microbiol 78(18):6749-6758

Griffiths RI, Thomson BC, James P, Bell T, Bailey M, Whiteley AS (2011) The bacterial biogeography of British soils. Environ Microbiol 13(6):1642-1654

Hanson CA, Fuhrman JA, Horner-Devine MC, Martiny JB (2012) Beyond biogeographic patterns: processes shaping the microbial landscape. Nat Rev Microbiol 10(7):497-506

Holmes DE, Finneran KT, O'neil RA, Lovley DR (2002) Enrichment of members of the family Geobacteraceae associated with stimulation of dissimilatory metal reduction in uranium-contaminated aquifer sediments. Appl Environ Microbiol (AEM) 68(5):2300-2306

Hori T, Müller A, Igarashi Y, Conrad R, Friedrich MW (2010) Identification of iron-reducing microorganisms in anoxic rice paddy soil by ${ }^{13} \mathrm{C}$-acetate probing. ISME J 4(2):267-278

Hori T, Aoyagi T, Itoh H, Narihiro T, Oikawa A, Suzuki K, Ogata A, Friedrich MW, Conrad R, Kamagata Y (2015) Isolation of microorganisms involved in reduction of crystalline iron (III) oxides in natural environments. Front Microbiol 6:386

Jäckel U, Russo S, Schnell S (2005) Enhanced iron reduction by iron supplement: a strategy to reduce methane emission from paddies. Soil Biol Biochem 37(11):2150-2154

Jiang Y, Liang Y, Li C, Wang F, Sui Y, Suvannang N, Zhou J, Sun B (2016) Crop rotations alter bacterial and fungal diversity in paddy soils across East Asia. Soil Biol Biochem 95:250-261

Kato S, Nakamura R, Kai F, Watanabe K, Hashimoto K (2010) Respiratory interactions of soil bacteria with (semi) conductive iron-oxide minerals. Environ Microbiol 12:3114-3123

Lalonde K, Mucci A, Ouellet A, Gélinas Y (2012) Preservation of organic matter in sediments promoted by iron. Nature 483(7388):198-200

Lauber CL, Hamady M, Knight R, Fierer N (2009) Pyrosequencingbased assessment of soil $\mathrm{pH}$ as a predictor of soil bacterial community structure at the continental scale. Appl Environ Microbiol 75(15):5111-5120

Li H, Peng J, Weber KA, Zhu Y (2011) Phylogenetic diversity of Fe (III)reducing microorganisms in rice paddy soil: enrichment cultures with different short-chain fatty acids as electron donors. J Soils Sediments 11(7):1234-1242
Liu J, Sui Y, Yu Z, Shi Y, Chu H, Jin J, Liu X, Wang G (2014) High throughput sequencing analysis of biogeographical distribution of bacterial communities in the black soils of northeast China. Soil Biol Biochem 70:113-122

Liu J, Sui Y, Yu Z, Shi Y, Chu H, Jin J, Liu X, Wang G (2015) Soil carbon content drives the biogeographical distribution of fungal communities in the black soil zone of northeast China. Soil Biol Biochem 83:29-39

Lovley DR (1987) Organic matter mineralization with the reduction of ferric iron: a review. Geomicrobiol J 5(3-4):375-399

Lovley DR, Holmes DE, Nevin KP (2004) Dissimilatory Fe (iii) and Mn (iv) reduction. Adv Microb Physiol 49:219-286

Mejia J, Roden EE, Ginder-Vogel MA (2016) Influence of oxygen and nitrate on $\mathrm{Fe}$ (hydr) oxide mineral transformation and soil microbial communities during redox cycling. Environ Sci Technol 50:3580-3588

Melton ED, Swanner ED, Behrens S, Schmidt C, Kappler A (2014) The interplay of microbially mediated and abiotic reactions in the biogeochemical Fe cycle. Nat Rev Microbiol 12(12):797-808

Pierra M, Carmona-Martínez AA, Trably E, Godon J-J, Bernet N (2015) Microbial characterization of anode-respiring bacteria within biofilms developed from cultures previously enriched in dissimilatory metal-reducing bacteria. Bioresour Technol 195:283-287

Reed DC, Algar CK, Huber JA, Dick GJ (2014) Gene-centric approach to integrating environmental genomics and biogeochemical models. Proc Natl Acad Sci U S A 111(5):1879-1884

Rodrigues JL, Pellizari VH, Mueller R, Baek K, Jesus EC, Paula FS, Mirza B, Hamaoui GS, Tsai SM, Feigl B (2013) Conversion of the Amazon rainforest to agriculture results in biotic homogenization of soil bacterial communities. Proc Natl Acad Sci U S A 110(3):988-993

Snoeyenbos-West O, Nevin K, Anderson R, Lovley D (2000) Enrichment of Geobacter species in response to stimulation of Fe (III) reduction in sandy aquifer sediments. Microb Ecol 39(2):153-167

Stumm W, Sulzberger B (1992) The cycling of iron in natural environments: considerations based on laboratory studies of heterogeneous redox processes. Geochim Cosmochim Acta 56(8):3233-3257

Wang N, Chen Z, Li H-B, Su J-Q, Zhao F, Zhu Y-G (2015) Bacterial community composition at anodes of microbial fuel cells for paddy soils: the effects of soil properties. J Soils Sediments 15(4):926-936

Weber KA, Achenbach LA, Coates JD (2006) Microbes pumping iron: anaerobic microbial iron oxidation and reduction. Nat Rev Microbiol 4:752-764

Yi W, You J, Zhu C, Wang B, Qu D (2013) Diversity, dynamic and abundance of Geobacteraceae species in paddy soil following slurry incubation. Eur J Soil Biol 56:11-18

Zhang S, Zhao F, Sun G, Su J, Yang X, Li H, Zhu Y (2015) Diversity and Abundance of Arsenic Biotransformation Genes in Paddy Soils from Southern China. Environ Sci Technol 49: 4138-4146 